\title{
Calculation of Normalized Pinning Force and Nature of Pinning Mechanism for Nano-Al Doped $\mathrm{MgB}_{2}$ Superconductor
}

\author{
Intikhab A. Ansari \\ Department of General Studies, Jubail Industrial College, P. O. Box - 10099, \\ Jubail Industrial City-31961, Saudi Arabia. \\ * Corresponding author email: intikhabansari@yahoo.com
}

Received: 31 January 2017 / Accepted: 2 February 2017 / Published: 3 February 2017

\begin{abstract}
The $J_{\mathrm{c}}(H)$ of nano-Al doped $\mathrm{MgB}_{2}$ samples has been calculated from $M(H)$ loop measurements at different temperatures. Normalized volume pinning forces as a function of the reduced field have been analyzed at different temperatures and doping level which was taken from the $J_{\mathrm{c}}(H)$ data. The modified scaling law was used as discussed by the Eisterer to analyze the pinning forces. This law was compared with the scaling law used by the Fietz and co-worker. The grain boundary pinning is found the dominant pinning mechanism in all the doped samples and exhibit the scaling behavior. The XRD and temperature dependence of resistivity confirms the successful substitution of nano- $\mathrm{Al}$ at $\mathrm{Mg}$ sites. The results endorse that the magnetic anisotropy decreased from pure to the dirty limit with the doping of the nano-Al. The enhancement in the $J_{\mathrm{c}}(H)$ of the sample with the nano-Al doping is due to decrease in the anisotropy and increase in the volume pinning forces. The $2 \%$ nano-Al doped sample between 15-30 K shows the highest $J_{\mathrm{c}}(H)$ among all the samples.
\end{abstract}

Keywords: Pinning force density; Critical current; $\mathrm{MgB}_{2}$; $\mathrm{Nano}-\mathrm{Al}$ doping

\section{Introduction}

In the last decades, the $\mathrm{MgB}_{2}$ demonstrates itself as a promising candidate for the practical applications due to its simple crystal structure, large coherence length, relatively high-temperature $\left(T_{\mathrm{c}}\right)$ and economical cost. However, the high critical current $\left(J_{\mathrm{c}}\right)$ of the bulk materials in magnetic field plays an important role in high field industrial applications [1]. The existence of high $J_{\mathrm{c}}$ up to $10^{6} \mathrm{~A} / \mathrm{cm}^{2}$ has been reported in the lots of literature. The value of $J_{\mathrm{c}}$ in pure material abruptly decreases in the presence of a high magnetic field. The effective improvement in $J_{\mathrm{c}}$ has been noticed due to the increase of pinning centers served by grain boundaries. Enormous efforts have been accustomed in order to enhance the $J_{\mathrm{c}}$ under the magnetic field [2]-[8]. The nanoparticle doping is one of the methods to improve the poor grain connection in pure $\mathrm{MgB}_{2}$. Introducing more pinning centers in the pristine $\mathrm{MgB}_{2}$ sample, exclusively nano size inclusions, generally provides a strong pinning force. The magnitude of bulk pinning force density, $F_{\mathrm{p}}$ can be calculated by the critical current density and an applied field, $J_{\mathrm{c}} \times H$. The $F_{p}$ was recently used to analyze the nature of pinning behavior in the nano- $\mathrm{Zn}$ doped $\mathrm{MgB}_{2}$ sample [9]. The $F_{\mathrm{p}}$ in the superconductor can be evaluated from the magnetization measurement at a particular temperature and is managed by the presence of impurities and microstructure of the materials. Fietz and co-worker [10] proposed the following scaling law for the normalized pinning force density $f \propto b^{p}(1-b)^{q}$, where $f=F_{\mathrm{p}}(b)=F_{\mathrm{p}} / F_{\mathrm{p}, \max }$ is the normalized pinning force density, $F_{\mathrm{p}, \max }$ is the maximum pinning force density, $b=H / H_{\text {irr }}$ is the reduced field and $H_{\text {irr }}$ is the irreversibility field where $J_{\mathrm{c}}$ practically becomes zero. The function $F_{\mathrm{p}}(b)$ shows a 
maximum value at $b_{\text {peak }}=p /(p+q)$. The exponents $p$ and $q$ are the characteristic for the prevailing pinning mechanism. Instantly, $p$ is predicted to be $1 / 2$ for grain boundary pinning or 1 for normal point pinning, while $q$ is same in both cases in this report. The study of the normalized volume pinning force as a function of field gives us the chance to know the behavior of the pinning mechanism in an individual case [11]. However, the $\mathrm{MgB}_{2}$ retains an intrinsic anisotropy in the presence of magnetic field [12], [13], the supercurrents never flow consistently because of the grain disorientation distribution on a sample surface. The clean grain boundaries not only drift the current in $\mathrm{MgB}_{2}$ [1] but also behave as effective pinning centers [14] and permit high currents in un-textured polycrystalline samples. Furthermore, secondary phases partly obstruct the current flow over the surface of the grains [15] and diminish the actual area over which the super-currents flow [16]. This problem is elaborated by the voids and secondary phases present over the surface of the grains that turn to inflate the current percolation, which is a usually inevitable result of the magnetic anisotropy [17].

The present study described the detailed analysis of the $J_{\mathrm{c}}$ and normalized pinning force, $f$ as a function of magnetic field and temperature for nano $\mathrm{Al}$ doped $\mathrm{MgB}_{2}$ sample using the simple scaling law described above. The difference between the previous report [6] and present is that in the prior report only magnetic behavior have been analyzed but in the present report I have calculated the $J_{\mathrm{c}}$ as well as the pinning mechanism for nano $\mathrm{Al}$ doped $\mathrm{MgB}_{2}$. In order to examine the dominant pinning mechanism for nano $\mathrm{Al}$ doped $\mathrm{MgB}_{2}$ sample this scaling law describes the significant results. In addition, it is shown that peak in reduced field dependence of the normalized pinning force is extensively shifted to the lower field by the anisotropy. The nano-Al doping may also affect the grain growth, thus leading to an improvement in the $J_{\mathrm{c}}$.

\section{Experimental Details}

$\mathrm{Mg}_{1-x} \mathrm{Al}_{x} \mathrm{~B}_{2}$ with different doping levels $(x=0,2$, 4 , and 6 at. \%) were synthesized by in-situ standard solid state reaction method under high vacuum as described elsewhere in detail [6]. The starting material powders (purity $>99 \%$ ) were mixed in the stoichiometric ratio supplied by SigmaAldrich. Here, the source of the nano-Al powder was nano- $\mathrm{Al}_{2} \mathrm{O}_{3}$. Finally, the bulk polycrystalline samples of the nano-Al doped $\mathrm{MgB}_{2}$ were achieved. The phase components were identified by the powder X-ray diffraction (XRD) measurement using $\mathrm{Cu} \mathrm{K}_{\alpha}$ radiation. The lattice parameters were calculated from the peak positions of the XRD plots. The transition temperature, $T_{\mathrm{c}}$ of the samples was determined by the temperature dependence of resistivity measurement using four-probe technique. The critical current density, $J_{\mathrm{c}}$ values were deduced from the hysteresis loop $M(H)$ using Bean's model. The DC magnetizations of these samples were measured using a PAR-4500 Vibrating Sample Magnetometer (VSM).

\section{Results and Discussion}

The x-ray diffraction (XRD) pattern of the doped and undoped $\mathrm{MgB}_{2}$ samples with different nano Al-concentration $(0 \%, 2 \%, 4 \%$ and $6 \%)$ is shown in Figure 1.

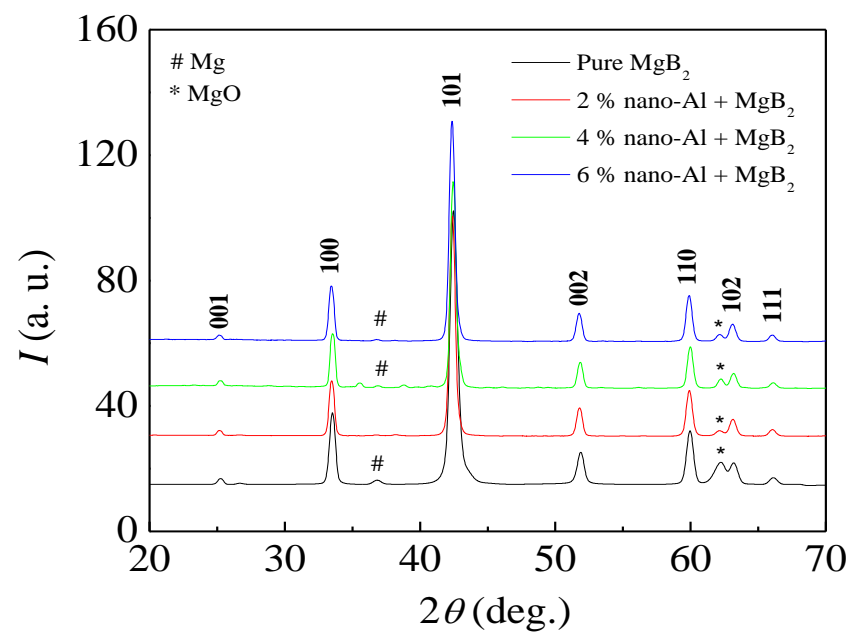

Figure 1: XRD pattern of $0 \%, 2 \%, 4 \%$ and $6 \%$ nano-Al doped $\mathrm{MgB}_{2}$ (Color figure online)

All the sample shows the $\mathrm{MgB}_{2}$ as the dominant phase with minor impurity of $\mathrm{MgO}$ and un-reacted $\mathrm{Mg}$. The Figure 1 of the present study indicates that $\mathrm{MgO}$ phase gradually decreased with the increasing doping level except the 6\% sample. Thus, it concludes that the oxygen which comes from dopant is effectively removed during a powder mixing process. The value of $a$ lattice parameter as well as $c$ lattice parameter decreased with the increase of the nano $\mathrm{Al}$ doping 
I A Ansari, J. Mod. Mater.; Vol. 3, Issue 1, pp: 33-40, 2017

concentration. The $a$ lattice parameter decreased from 3.087-3.081 $\AA$ on the other hand the $c$ lattice parameter suppressed from 3.524-3.350 $\AA$. It is evident from these results that nano $\mathrm{Al}$ atoms partially occupied the sites of $\mathrm{Mg}$ in the $\mathrm{MgB}_{2}$ matrix. It is furthermore necessary to study the effect of nano $\mathrm{Al}$ dopant on the temperature dependence of resistivity which is described in the Figure 2.

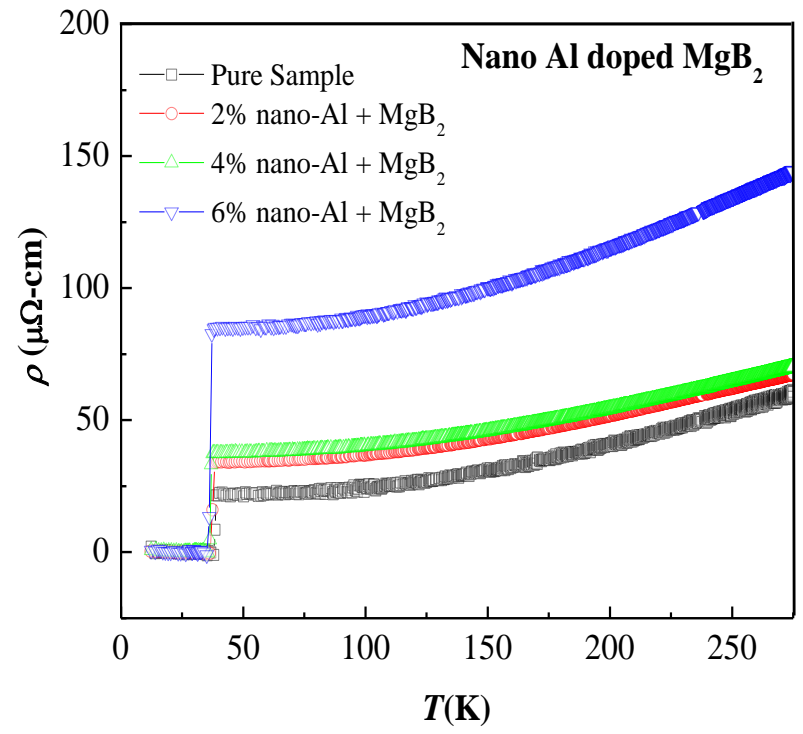

Figure 2: Temperature dependence of the resistivity of the nano-Al doped $\mathrm{MgB}_{2}$ samples.

It is remarkable that the normal-state resistivities of all the samples are almost the same except the $6 \%$ doped sample. The later might be distinct due to the formation of dominant impurity phase $\mathrm{MgO}$ at grain boundaries in this doped sample. The residual resistivity ratio (RRR) values shows the reduction from 3-1.82 with the increase of doping level. The temperature dependence of electrical resistivity of all the samples helps in order to understand the electron-phonon interaction, which is described by the BlochGruneisen integral [18]. The nano-Al substitution is further supported from the change in the $T_{\mathrm{c}}$ of the doped samples as shown in the Table 1.

Table 1: Variation of a-lattice parameter, $c$-lattice parameter and transition temperature $T_{c}$ with the nano-Al doped $\mathrm{MgB}_{2}$

\begin{tabular}{|c|c|c|c|}
\hline $\boldsymbol{x}(\boldsymbol{\%})$ & $\boldsymbol{a}(\mathbf{\AA})$ & $\boldsymbol{c}(\mathbf{\AA})$ & $\boldsymbol{T}_{\mathbf{c}}(\mathbf{K})$ \\
\hline 0.0 & 3.087 & 3.524 & 38 \\
\hline 2.0 & 3.085 & 3.522 & 36 \\
\hline 4.0 & 3.083 & 3.351 & 36 \\
\hline 6.0 & 3.081 & 3.350 & 35 \\
\hline
\end{tabular}

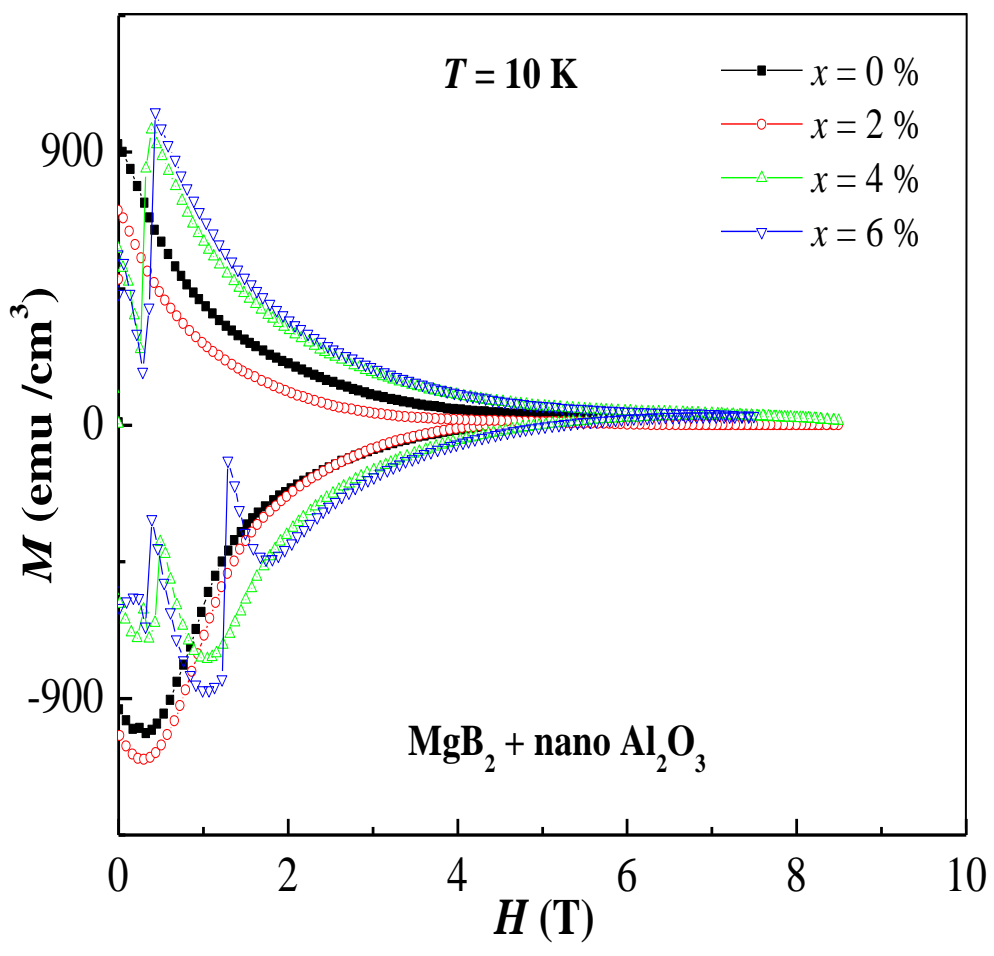

Figure 3: Hysteresis loops of all the samples at 10 $K$. Avalanches are observed at low doping levels (Color figure online)

Figure 3 shows the hysteresis loops of all the samples at $10 \mathrm{~K}$ with a DC field. It is clear that the pure sample shows continuous hysteresis loop while the doped samples show sharp flux jumps below 2T. As shown in Figure 3 at 10K, the 6\% sample exhibit the better $M(\mathrm{H})$ behavior among all the samples. Zhao et al. [19] described that using a SQUID for magnetization measurements, avalanches vanish due to the very low speed of data acquisition. In order to further find out evidences, the magnetic field dependence of $J_{\mathrm{c}}$ at different temperature has been calculated under different doping level.

Figure 4 shows the magnetic field dependence of $J_{\mathrm{c}}$ at $10,15,20$ and $30 \mathrm{~K}$ for pure and nano Aldoped $\mathrm{MgB}_{2}$ samples. It is evident from the figure that the value of $J_{\mathrm{c}}$ is gradually improved of $2 \%$ doped sample in the temperature range from 15 $30 \mathrm{~K}$. At $10 \mathrm{~K}$ temperature, the $6 \%$ sample exhibit the better $J_{\mathrm{c}}(\mathrm{H})$ behavior among all the four samples. Furthermore, at $15 \mathrm{~K}$ temperature, all the doped sample shows relatively same $J_{\mathrm{c}}$ value at lower field but when the field enhances the difference in $J_{\mathrm{c}}$ becomes comparatively enough as the prior. The $2 \%$ nano-Al doped sample offered the highest $J_{\mathrm{c}}$ at $15-30 \mathrm{~K}$ but not at $10 \mathrm{~K}$ because 
at lower temperatures $(<15 \mathrm{~K})$, there might be some kind of cross-over taken place in the $2 \%$ sample. On the contrary, the pinning strength is a function of the temperature. For $2 \%$ sample, at lower temperature $(<15 \mathrm{~K})$, the pinning is higher and at higher temperature $(>15 \mathrm{~K})$ the pinning strength becomes weak and actual grain connectivity plays the role of carrying the current. This explains the cross-over of $J_{\mathrm{c}}$ plot around 15 $\mathrm{K}$ for $2 \%$ nano-Al doped sample. Due to the flux avalanches (instabilities) in $M(H)$ plot (some of them are reported elsewhere [6] ), there are some jumps reflected at $10 \mathrm{~K}$ (nearby $1 \mathrm{~T}$ ) and $20 \mathrm{~K}$ (nearby $3.5 \mathrm{~T}$ ) for $6 \%$ doped and pure sample, respectively as shown in the Figure 4. In addition, at $30 \mathrm{~K}$ temperature the $4 \%$ and $6 \% \mathrm{Al}$ doped sample depicts some jumps as well close to $0.25 \mathrm{~T}$ field. There are two kinds of avalanches in superconductors, as pointed out by Altshuler and Johansen [20]. The first one refers to the dynamical driven avalanches, i.e., small isothermal avalanches related to the evolution of the critical state model. The second type refers to thermally driven avalanches, also called flux avalanches, and they are related to thermomagnetic instabilities, leading to a sudden penetration of flux in an approximately adiabatic process, as discussed by Mints and Rakhmanov [21]. One of the signatures of the flux avalanches is a jumpy behavior in $M(H)$ curves, which named these phenomena as flux jumps. In order to further find out evidences, the normalized pinning force has been calculated as a function of reduced field under different doping level and temperature. It is remarkable that the critical field $J_{\mathrm{c}}$ is directly proportional to the $F_{\mathrm{p}, \max }$.

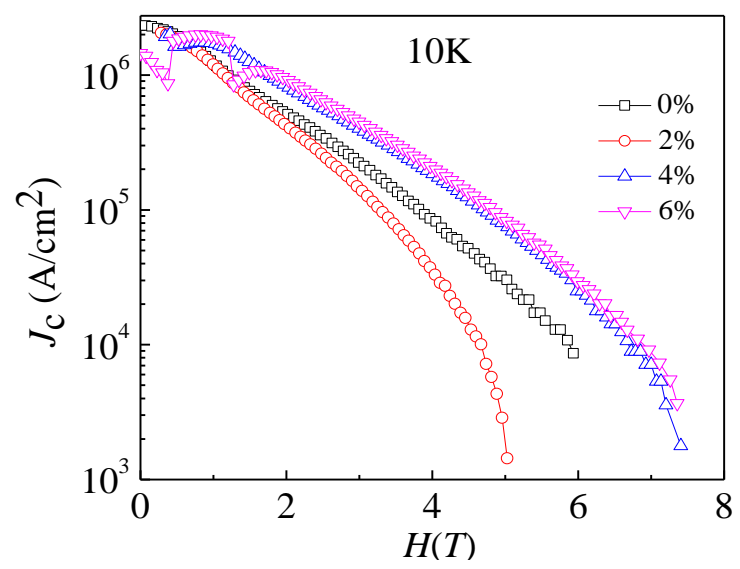

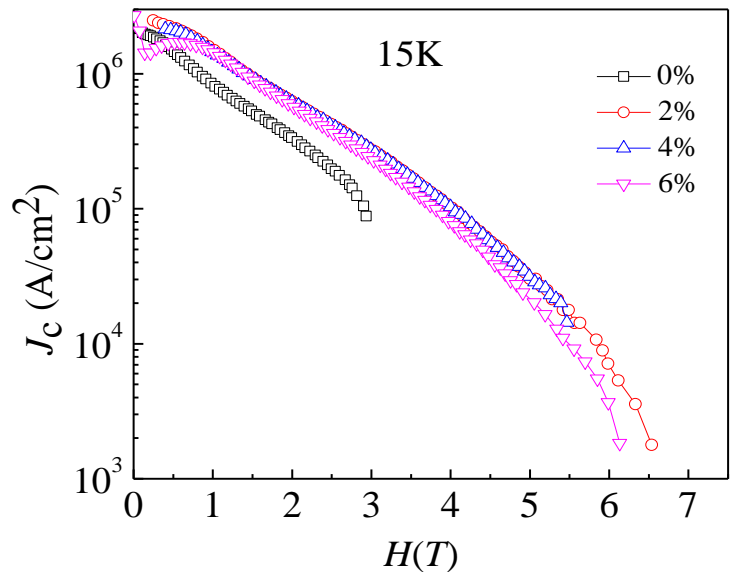
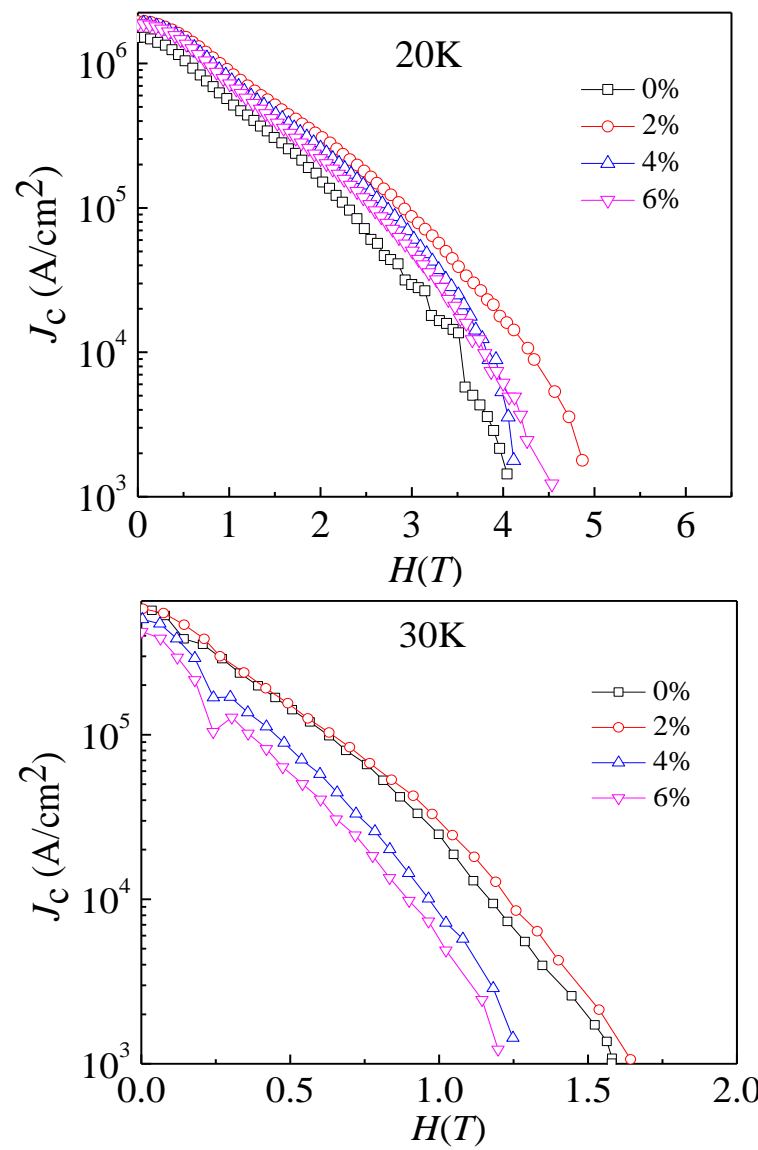

Figure 4: Magnetic field dependence of $J_{c}$ at difference temperatures for $M g_{1-x} A l_{x} B_{2}$ (Color figure online)

In order to study the characteristics of the pinning mechanism for different $\mathrm{Al}$ doped $\mathrm{MgB}_{2}$ samples, the normalized volume pinning force $\left(F_{\mathrm{p}} / F_{\mathrm{p}, \max }\right)$ as a function of reduced field $\left(H / H_{\text {irr }}\right)$ has been analyzed at different temperatures. The left column of the Figure 5 has shown the outcomes of these samples. 
I A Ansari, J. Mod. Mater.; Vol. 3, Issue 1, pp: 33-40, 2017
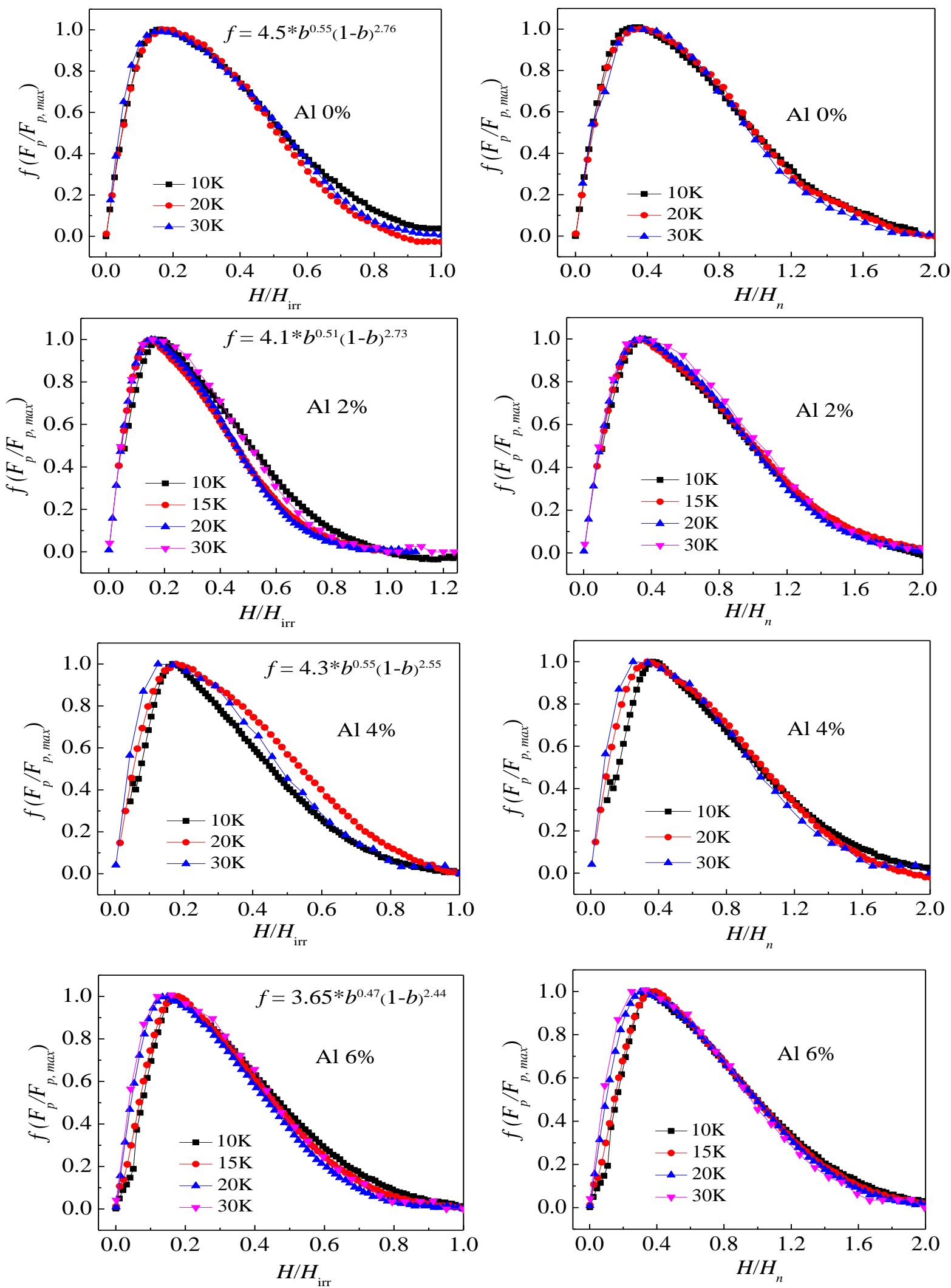

Figure 5: Normalized pinning force as a function of (left column) reduced field $\left(H / H_{\text {irr }}\right)$ and (right column) modified reduced field $\left(\mathrm{H} / \mathrm{H}_{n}\right)$. (Color figure online) 
Calculation of Normalized Pinning Force and Nature of Pinning Mechanism for Nano-Al Doped MgB ${ }_{2}$ Superconductor

Table 2: The value of $b_{\text {peak }}$ and $b_{\text {npeak }}$ for all samples at different temperature

\begin{tabular}{|c|l|l|l|l|l|l|l|l|}
\hline \multirow{2}{*}{$\boldsymbol{T}(\mathbf{K}) / \boldsymbol{x} \%$} & \multicolumn{3}{|c|}{$\mathbf{0 \%}$} & \multicolumn{2}{c|}{$2 \%$} & \multicolumn{2}{c|}{$4 \%$} & \multicolumn{2}{c|}{ 6\% } \\
\cline { 2 - 9 } & $b_{\text {peak }}$ & $b_{\text {npeak }}$ & $b_{\text {peak }}$ & $b_{\text {npeak }}$ & $b_{\text {peak }}$ & $b_{\text {npeak }}$ & $b_{\text {peak }}$ & $b_{\text {npeak }}$ \\
\hline 10 & 0.151 & 0.285 & 0.161 & 0.341 & 0.167 & 0.353 & 0.181 & 0.366 \\
\hline 15 & - & 0.348 & 0.157 & 0.349 & - & 0.358 & 0.179 & 0.391 \\
\hline 20 & 0.149 & 0.326 & 0.152 & 0.332 & 0.166 & 0.333 & 0.177 & 0.336 \\
\hline 30 & 0.119 & 0.249 & 0.121 & 0.250 & 0.124 & 0.312 & 0.157 & 0.328 \\
\hline
\end{tabular}

It is evident from the Figure 5 that the dominant pinning occurs in undoped, $2 \%$ and $6 \%$ nano $\mathrm{Al}$ doped samples at different temperatures and follow the scaling law $f \propto b^{p}(1-b)^{q}$. In case of undoped sample the values of $p$ and $q$ are observed to be 0.55 and 2.75 , respectively which is in agreement with the value of $p$ and $q$ for grain boundary pinning $(p=1 / 2$ and $q=2)$. In case of $2 \%$ and $6 \% \mathrm{Al}$ doped samples the values of $p$ are 0.51 and 0.47 while the values of $q$ are 2.73 and 2.44 , respectively. These values are still approaching to the grain boundary pinning. The $4 \%$ sample shows the different behavior at different temperatures and does not coincide at all. The values of the $p$ and $q$ are still close to the grain boundary pinning in case of $4 \%$ sample but the disperse might be due to the fluctuation in the $M(H)$ plot at lower $J_{\mathrm{c}}$ value.

To understand the in-depth effect of the nano-Al doped $\mathrm{MgB}_{2}$ on the nature of pinning, the peak position in $f\left(F_{\mathrm{p}} / F_{\mathrm{p}, \max }\right)$ vs. $H / H_{\text {irr }}$ plot has been taken into account which is indicated by $b_{\text {peak }}$ here. In the isotropic materials, the $F_{\max }$ occurs at $b_{\text {peak }}$ $=0.2$ for grain boundary pinning and $b_{\text {peak }}=0.33$ for point pinning. The shift of $b_{\text {peak }}$ of the normalized volume pinning force depends not only on the dominant pinning mechanism but also on the anisotropy $\gamma$ and on the percolation threshold $p_{\mathrm{c}}$. The effect of the anisotropy on scaling which generally gives the deviation of the peak position in the different pinning mechanism has been studied theoretically for pure $\mathrm{MgB}_{2}$ system as proposed by the Eisterer [22]. He reported that the significant anisotropic changes occurs due to the field dependence of the volume pinning force in the polycrystalline samples. In the pure $\mathrm{MgB}_{2}$, the value of $p_{\mathrm{c}}$ varies minutely ( 0.25 to $0.30)$ but the value of $\gamma$ alters between 1 (dirty limit) to 6 (clean limit). The maximum peak field value $\left(b_{\text {peak }}\right)$ is hyper-sensitive to the anisotropy $\gamma$ rather than percolation threshold $p_{\mathrm{c}}$. Therefore, analyzing the pinning behavior from the $b_{\text {peak }}$ only without prior knowledge of $\gamma$ and $p_{\mathrm{c}}$ is challenging using the scaling law where $b=H / H_{\text {irr }}$ is involved. To reduce this difficulty, Eisterer has proposed the modified scaling law to study the pinning mechanism in bulk samples in which anisotropy $\gamma$ and percolation threshold $p_{\mathrm{c}}$ has minor effect on peak field position. In this new law $f$ is plotted as a function of normalized field $b_{\text {npeak }}=H / H_{\mathrm{n}}$ where $H_{\mathrm{n}}$ is the field at which the pinning force becomes half of $F_{\mathrm{p}, \max }$ value. He theoretically explained that the peak position shifts very slightly, $3 \%$ only in case of grain boundary pinning $\left(b_{\text {npeak }}=0.341\right.$ to $0.352)$ and $6 \%$ in case of the point pinning $\left(b_{\text {npeak }}\right.$ $=0.465$ to 0.491 ) from clean to dirty sample respectively in both conditions. It is easily possible to distinguish between grain boundary pinning and point pinning from just the difference of peak field position (0.341 to 0.465$)$, which differ by nearly $36 \%$. The new scaling scheme for nano-Al doped $\mathrm{MgB}_{2}$ sample is mentioned in the right column of Figure 5, which articulates the better overlapping of the data acquisition for different temperatures. The values of $b_{\text {peak }}$ and $b_{\text {npeak }}$ which shows the peak position in $H / H_{\text {irr }}$ and $H / H_{\mathrm{n}}$ plot, respectively are exposed in Table 2 for different samples at different temperatures. Eisterer has revealed that $b_{\text {peak }}$ or $b_{\text {npeak }}$ shift towards lower field value as the anisotropy enhances. Due to large flux avalanche (instability) in $M(H)$ plot at $15 \mathrm{~K}$ in pure and $4 \%$ doped sample, the data is absent at $15 \mathrm{~K}$ in $f$ vs. reduced field plot for these samples. From Figure 5 and Table 2 as well, it is evident that the grain boundary pinning is the dominant pinning mechanism in all the four samples at different 
I A Ansari, J. Mod. Mater.; Vol. 3, Issue 1, pp: 33-40, 2017

temperature. Table 2 clearly shows the enhancement of $b_{\text {peak }}$ value in the Al-doped samples in comparison with pure sample. This represents that nano $\mathrm{Al}_{2} \mathrm{O}_{3}$ has decreased the magnetic anisotropy from pure to dirty limit. It is notable that as the $\mathrm{Al}$ doping increases the sample becomes less anisotropic, on the contrary the pure sample is highly anisotropic in nature.

\section{Conclusion}

With the help of hysteresis loop, the $J_{\mathrm{c}}(H)$ was calculated for all the doped and undoped samples at different temperatures. Among all the samples the $2 \%$ doped sample reveals the highest $J_{\mathrm{c}}(H)$ at all the temperatures except $10 \mathrm{~K}$. The normalized pinning force density as a function of reduced field was calculated by two scaling ways as discussed by the Fietz et al. and Eisterer. It was confirmed that all samples show grain boundary pinning. At last, the value of $b_{\text {peak }}$ and $b_{\text {npeak }}$ for all samples at different temperatures has been tabulated. The increase in the value of the $b_{\text {peak }}$ with the nano $\mathrm{Al}$ doping denotes that the doping decreases the anisotropy of the samples. These $b_{\text {peak }}$ values are responsible for grain boundary pinning. Furthermore, it can be concluded that the suppression in the anisotropy is partially accountable for the enhancement of the $J_{\mathrm{c}}(H)$ values.

\section{Acknowledgments}

I would like to thank Jafar Parakkandy (KSU, Riyadh) for experimental ends. This work is supported by the General Studies Department, Jubail Industrial College, Royal Commission in Jubail, Saudi Arabia under contract no. 2101349345.

\section{How to Cite this Article:}

I. Ansari, "Calculation of Normalized Pinning Force and Nature of Pinning Mechanism for Nano-Al Doped $\mathrm{MgB}_{2}$ Superconductor", J. Mod. Mater., vol. 3, no. 1, pp. 33-40, Feb. 2017. doi: $10.21467 / \mathrm{jmm} \cdot 3 \cdot 1 \cdot 33-40$

\section{References}

[1] D. Larbalestier, A. Gurevich, D. M. Feldmann, and A. Polyanskii, "High-Tc superconducting materials for electric power applications," Nature, vol. 414, no. 6861, pp. 368-377, Nov. 2001.

[2] M. A. Susner et al., "Influence of $\mathrm{Mg} / \mathrm{B}$ ratio and $\mathrm{SiC}$ doping on microstructure and high field transport Jc in MgB2 strands," Phys. C Supercond., vol. 456, no. 1, pp. 180-187, 2007.

[3] M. Putti, M. Affronte, C. Ferdeghini, P. Manfrinetti, C. Tarantini, and E. Lehmann, "Observation of the Crossover from Two-Gap to Single-Gap Superconductivity through Specific Heat Measurements in Neutron-Irradiated $\mathrm{MgB}_{2}$," Phys. Rev. Lett., vol. 96, no. 7, p. 77003, Feb. 2006.

[4] A. Gümbel, J. Eckert, G. Fuchs, K. Nenkov, K.-H. Müller, and L. Schultz, "Improved superconducting properties in nanocrystalline bulk $\mathrm{MgB}_{2}$, " Appl. Phys. Lett., vol. 80, no. 15, pp. 2725-2727, Apr. 2002.

K. P. Singh et al., "Nano $\mathrm{Fe}_{3} \mathrm{O}_{4}$ Induced Fluxoid Jumps and Low Field Enhanced Critical Current Density in $\mathrm{MgB} 2$ Superconductor," J. Supercond. Nov. Magn., vol. 21, no. 1, pp. 39-44, Jan. 2008.

[6] I. A. Ansari et al., "The effect of nano-alumina on structural and magnetic properties of $\mathrm{MgB}_{2}$ superconductors," Supercond. Sci. Technol., vol. 20, no. 8, pp. 827-831, Aug. 2007.

[7] S. X. Dou et al., "Enhancement of the critical current density and flux pinning of $\mathrm{MgB}_{2}$ superconductor by nanoparticle SiC doping," Appl. Phys. Lett., vol. 81, no. 18, pp. 3419-3421, Oct. 2002.

[8] H. Yamada, M. Hirakawa, H. Kumakura, A. Matsumoto, and H. Kitaguchi, "Critical current densities of powder-in-tube $\mathrm{MgB}_{2}$ tapes fabricated with nanometer-size Mg powder," Appl. Phys. Lett., vol. 84, no. 10, pp. 1728-1730, Mar. 2004.

[9] M. Shahabuddin, I. A. Ansari, N. S. Alzayed, K. A. Ziq, and A. F. Salem, "Effect of Nano ZnO Doping on the Nature of Pinning of $\mathrm{MgB}_{2}$ Superconductors," $J$. Supercond. Nov. Magn., vol. 26, no. 5, pp. 1547-1552, May 2013

[10] W. A. Fietz and W. W. Webb, "Hysteresis in Superconducting Alloys-Temperature and Field Dependence of Dislocation Pinning in Niobium Alloys," Phys. Rev., vol. 178, no. 2, pp. 657-667, Feb. 1969.

[11] D. Dew-Hughes, "The role of grain boundaries in determining $J_{\mathrm{c}}$ in high-field high-current superconductors," Philos. Mag. Part B, vol. 55, no. 4, pp. 459-479, Apr. 1987.

[12] M. Zehetmayer, M. Eisterer, J. Jun, S. M. Kazakov, J. Karpinski, and H. W. Weber, "Magnetic field dependence of the reversible mixed-state properties of superconducting $\mathrm{MgB}_{2}$ single crystals and the influence of artificial defects," Phys. Rev. B, vol. 70, no. 21, p. 214516, Dec. 2004.

[13] L. Lyard et al., "Anisotropies of the Lower and Upper Critical Fields in $\mathrm{M} \mathrm{g} \mathrm{B}_{2}$ Single Crystals," Phys. Rev. Lett., vol. 92, no. 5, p. 57001, Feb. 2004.

[14] E. Martínez, P. Mikheenko, M. Martínez-López, A. Millán, A. Bevan, and J. S. Abell, "Flux pinning force in bulk $\mathrm{Mg} \mathrm{B}_{2}$ with variable grain size," Phys. Rev. $B$, vol. 75, no. 13, p. 134515, Apr. 2007.

[15] R. F. Klie, J. C. Idrobo, N. D. Browning, K. A. Regan, N. S. Rogado, and R. J. Cava, "Direct observation of nanometer-scale $\mathrm{Mg}$ - and $\mathrm{B}$-oxide phases at grain boundaries in $\mathrm{MgB}_{2}$," Appl. Phys. Lett., vol. 79, no. 12, pp. 1837-1839, Sep. 2001.

[16] J. M. Rowell et al., "The widely variable resistivity of $\mathrm{MgB}_{2}$ samples," Supercond. Sci. Technol., vol. 16, no. 6, pp. R17-R27, Jun. 2003.

[17] M. Eisterer et al., "Magnetic properties and critical currents of $\mathrm{MgB}_{2}$," Supercond. Sci. Technol., vol. 20, no. 12, pp. R47-R73, Dec. 2007. 
Calculation of Normalized Pinning Force and Nature of Pinning Mechanism for Nano-Al Doped $\mathrm{MgB}_{2}$ Superconductor

[18] I. A. Ansari, "Numerical solution of Bloch-Gruneisen function to determine the contribution of electronphonon interaction in polycrystalline $\mathrm{MgB}_{2}$ superconductor," Phys. C Supercond., vol. 470, no. 11, pp. 508-510, 2010

[19] Z. W. Zhao et al., "Suppression of superconducting critical current density by small flux jumps in $\mathrm{MgB}_{2}$ thin films," Phys. Rev. B, vol. 65, no. 6, p. 64512, Jan. 2002.

[20] E. Altshuler and T. H. Johansen, "Colloquium:

\section{Publish your research article in AIJR journals-}

$\checkmark \quad$ Online Submission and Tracking

$\checkmark$ Peer-Reviewed

$\checkmark$ Rapid decision

$\checkmark$ Immediate Publication after acceptance

$\checkmark \quad$ Articles freely available online

$\checkmark \quad$ Retain full copyright of your article.

Submit your article at journals.aijr.in
Experiments in vortex avalanches," Rev. Mod. Phys., vol. 76, no. 2, pp. 471-487, Apr. 2004.

[21] R. G. Mints and A. L. Rakhmanov, "Critical state stability in type-II superconductors and superconducting-normal-metal composites," Rev. Mod. Phys., vol. 53, no. 3, pp. 551-592, Jul. 1981.

[22] M. Eisterer, "Calculation of the volume pinning force in $\mathrm{MgB}_{2}$ superconductors," Phys. Rev. B, vol. 77, no. 14, p. 144524, Apr. 2008.

Publish your books with AIJR publisher-

$\checkmark \quad$ Publish with ISBN and DOI.

$\checkmark$ Publish Thesis/Dissertation as Monograph.

$\checkmark$ Publish Book Monograph.

$\checkmark$ Publish Edited Volume/ Book.

$\checkmark$ Publish Conference Proceedings

$\checkmark \quad$ Retain full copyright of your books.

Submit your manuscript at books.aijr.org 